A high-speed photomultiplier gating circuit for luminescence measurements

Thomas M. YoshidaThomas M. JovinB. George Barisas

Citation: 60, (1989); doi: 10.1063/1.1140628

View online: http://dx.doi.org/10.1063/1.1140628

View Table of Contents: http://aip.scitation.org/toc/rsi/60/9

Published by the American Institute of Physics 


\title{
A high-speed photomultiplier gating circuit for luminescence measurements
}

Thomas M. Yoshida

Colorado State University, Department of Chemistry, Fort Collins, Colorado 80523

Thomas M. Jovin

Max Planck Institut für Biophysikalische Chemie, Abteilung Molekulare Biologie D-3400, Göttingen, Federal Republic of Germany

B. George Barisas ${ }^{2)}$

Colorado State University, Department of Chemistry, Fort Collins, Colorado 80523

(Received 3 April 1989; accepted for pubication 30 May 1989)

\begin{abstract}
A high-speed, normally on, gating circuit has been developed for linear-focused photomultipliers such as the EMI 9816 . The gate is capable of attenuating photomultiplier response by $>5000: 1$ with a turn-off and turn-on time of 60 and $40 \mathrm{~ns}$, respectively. The circuit is compact enough to fit in a standard photomultiplier housing and has low enough power consumption to run off the photomultiplier high-voltage supply. The high speed and high extinction efficiency make this gate suitable for pulse rejection in low-light level, submicrosecond, time-resolved luminescence measurements.
\end{abstract}

\section{INTRODUCTION}

Numerous time-resolved spectroscopic techniques involve exciting a sample with a relatively intense pulse of light and then probing a time-dependent optical signal such as phosphorescence, ${ }^{1}$ fluorescence, ${ }^{2}$ or fuorescence depletion ${ }^{3.4}$ with a photomultiplier tube (PMT) for several milliseconds following the pulse. Luminescence and scattered light Guring the pulse interval can be thousands of times greater than during the subsequent optical signal. Under such circumstances serious PMT saturation artifacts, such as persistent anode currents and gain saturation, ${ }^{5,6}$ may interfere with measurements directly following the excitation pulse. In extreme cases sputtering of the active material on the photocathode and dynodes will eventually result in permanent tube damage. These problems arcompounded if the PMT is already operating near saturation when the high-intensity pulse occurs, as in the case with some fluorescence depletion measurements. ${ }^{3,4}$ Pulse rejection by PMT gating is thereforc essential to eliminate these problems and allow measurement of time-dependent kinetic decay processes which occur microseconds to milliseconds after an excitation pulse. Since pulse-generating devices (for example, acousto-optic modulators, Pockels cells, and pulsed lasers) generally have fast response times ( $<10 \mathrm{~ns}$ ), PMT gating speed often limits the time resolution of the instrument. Also, requirements of PMT circuit and housing design often mandate that space and power consumption be minimized. Therefore, highspeed, simple, and efficient gating circuitry must be designed to meet these requirements.

Various methods have been used to gate PMTs, including pulsing the high-voltage PMT power supply, ${ }^{7}$ pulsing the voltage of one or more dynodes, ${ }^{6,8.18}$ pulsing the photocathode potential, ${ }^{19}$ switching the potential of a focusing electrode, ${ }^{19-23}$ or switching a grid specially incorporated into the PMT for gating purposes. ${ }^{24}$ The method chosen is depen- dent on the PMT type, required mode (normally on or normally off), the required extinction efficiency, gating speed and repetition rate, and availability of a switchable grid or focusing electrode. Each method has its own advantages and disadvantages, which are discussed by Farinelli and Malvano $^{19}$ and Wardle. ${ }^{25}$

A popular photomultiplier used for luminescence measurements is the EMI 9816 (Thorn EMI Gencom Inc., Fairview, $\mathrm{NJ}$ ). The 9816 is a 14-stage, linear-focused PMT with an $\$-20$ spectral response. Because of its high gain and fast response times, this PMT is well suited for fast photoncounting applications. For low-light-level measurements the 9816 does have the disadvantage of a relatively large photocathode surface area (46- $\mathrm{mm}$ diameter), which may produce excessive dark counts when operated in the photoncounting mode. This problem can be remedied by placing various magnets (PR-411 Magnetic Defocusing Assembly, Products for Research Inc, Danvers, MA) directly in front of the PMT window. Two such magnets reduce the effective cathode diameter to $8 \mathrm{~mm}$, resulting in a decrease in the dark counts from 500 to $32 \mathrm{cps}$ at $-30^{\circ} \mathrm{C}^{3}$

Although commercial gating circuits are available for focused PMTs such as the 9816 (model GB1001A and GB1001B, Thorn EMI Gencom Inc.), 1,26 they are generally not fast enough for measurements of submicrosecond kinetics. The normally on GB1001A uses a single transistor to actively pull down the focusing electrode $-45 \mathrm{~V}$ relative to the photocathode, resulting in a relatively fast turn-of time of $200 \mathrm{~ns}$. Unfortunately, the focusing electrode is not actively pulled up to its normally on potential $200 \mathrm{~V}$ positive relative to the photocathode. This results in a slow turn-on time of $>1 \mu$ s.

Due to the popularity of the 9816 for appiications where gating and fast recovery of PMT gain following a gating pulse are necessary, we have developed a simple, high-speed, 


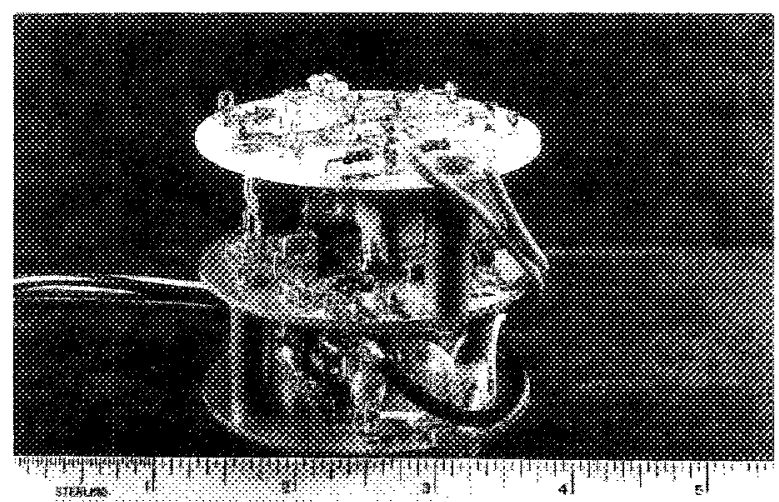

FiG. 1. Photograph of the high-speed photomultiplier gate. From top to bottom are the power, gate, and logic boards.

normally on gating circuit that has nanosecond response times and high extinction efficiencies. The entire circuit is compact enough to fit in a standard PMT housing and has low enough power consumption to run of a standard PMT high-voltage power supply. Under normal operating conditions the focusing electrode is held at the first dynode potential. In response to an external TTL gate input, the gating circuit switches the focusing electrode potential $100 \mathrm{~V}$ negative relative to the photocathode, sweeping photoelectrons away from the first dynode. Biasing the potential of the focusing electrode in this manner results in an extinction efficiency of $>5000: 1$ with a PMT turn-of time of $60 \mathrm{rrs}$ and a turn-on time of $40 \mathrm{~ns}$.

\section{CIRCUIT DESCRIPTION}

The gating circuit is based on a pair of complementary, high-voltage power MOSFETs (TMOS, Motorola Semiconductor Products Inc., Phoenix, AZ). These transistors are ideal for this type of application because of their high switching speed, high breakdown voltage ( $500 \mathrm{~V}$ for MTP2 250 and MTP2N50), small size, and high gate impedance. Because these are enhancement-mode devices and because of the voltage-sensitive gate input, very simple gate drive circuitry can be utilized resulting in a simple, compact circuit.

The gating circuit consists of three 2.5 -in.-diam printed circuit boards stacked so as to occupy a space $2.5 \mathrm{in}$. high (Fig. 1). The circuit is functionally divided into a power supply board, a low-voltage logic board, and a high-voltage gate board (Fig. 2). The logic and gate circuit boards have ground planes to reduce ringing and noise.

The power board provides the $+10-\mathrm{V}$ logic voltage, the MOSFET gate bias voltages ( $\mathrm{PB}$ and $\mathrm{NB}$ ), and the photocathode (PC) and first dynode (DY1) voltages. A chain of zener diodes is used to generate these voltages so that they are easily adjustable and independent of the changes in the HV input. Because of the low current through the zener diode chain, the manufacturer's voltage specifications are generally not accurate; therefore, individual diodes are selected based on on-board voltage measurements.

The logic board serves to isolate the TTL gate drive signal from the high-voltage switching circuit by way of an optocoupler. The negative-true gate input is referenced to chassis ground. The rest of the circuit is referenced to a floating ground ( $F G$ ), which is maintained near HV $(-1800$ V). The optocoupler will withstand maximum isolation voltages to $3000 \mathrm{~V}$, which sets the maximum value for the HV input. The output of the optocoupler goes to a CMOS inverter which drives a pair of bipolar transistors in a complementary emitter follower configuration. This stage provides a 10 $V$ gate drive capable of sinking and sourcing $>1 \mathrm{~A}$ to quickly charge and discharge the MOSFET gate capacitances of $500-1000 \mathrm{pr}$.

The gate board contains the high-voltage switching transistors along with components to bias the MOSFET gates. The gates are biased to maximize switching speed and

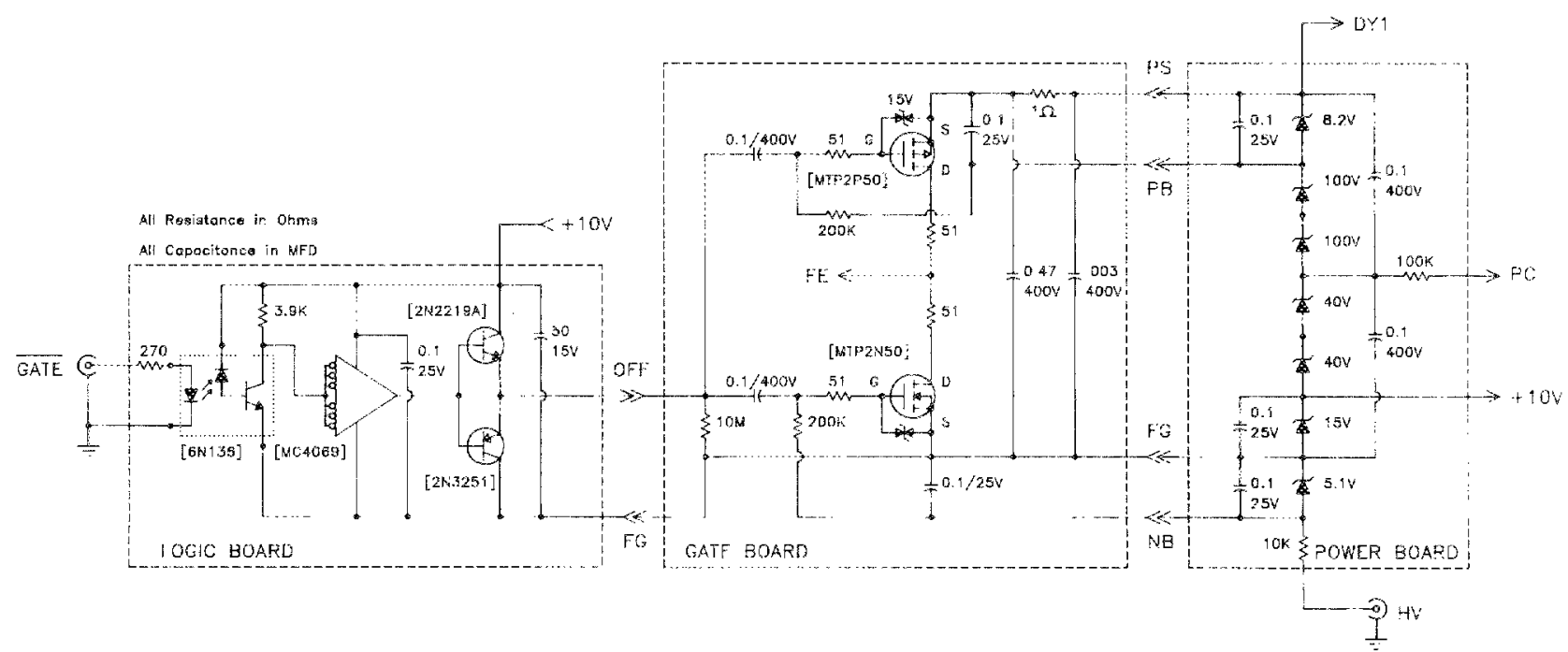

Fro. 2. Schematic diagram of the PMT gating circuit. All resistances are ohms and all capacitance are microfarads. PC is the photocathode output, DY 1 is the first dynode, PS is the $P$-channel source voltage, P'B is the P-channel gate bias, FE is the focusing electrode, FG is fleating ground, NB is the $N$-channel gate bias voltage, and HV is the PMT high-voltage input. The zener diodes are selected based on on-board voltage measurements. The values Eisted in the figure are the manufacturer's rating. The actual voltage drops are given is the text. 


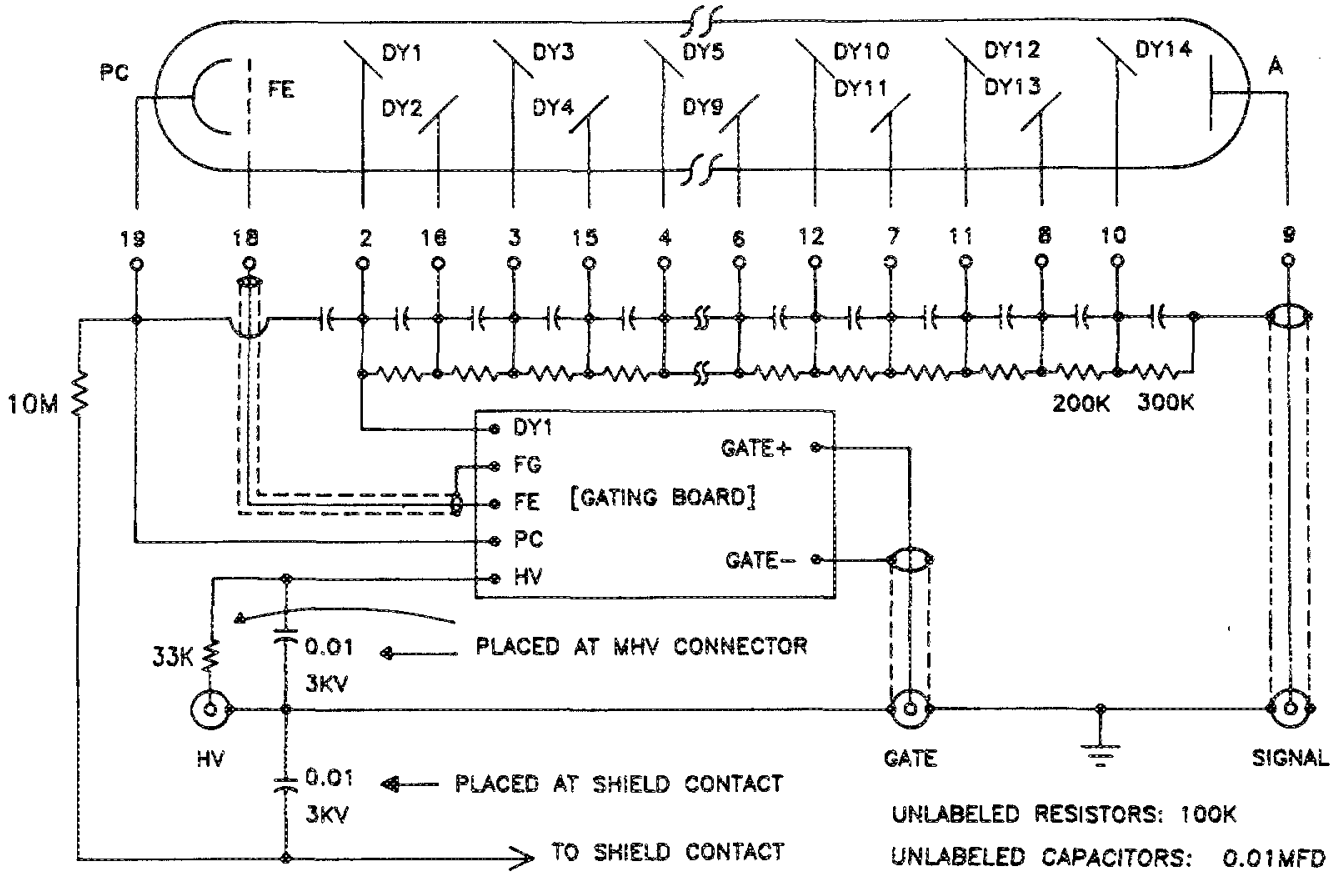

FIG. 3. Photomuitiplier divider network for the 9816 PMT showing the wiring of the gating circuit. The resistors between dynodes 13 and 14 and between 14 and ground, may be replaced with 200 - and 300 -V zener diodes, respectivefy, to improve PMT lincarity at high light levels as required.

to eliminate the possibility of both transistors conducting simultaneously, which would result in a temporary short circuit. The $P$-channel gate is biased so the initial gate-tosource voltage $V_{\mathrm{GS}}(P)$ is $-6 \mathrm{~V}$ and the $N$-channel gate is biased so $V_{G S}(N)=-4 \mathrm{~V}$. When biased this way the $p_{-}$ channel FET is conducting and the $N$-channel FET is nonconducting, and so the focusing electrode FE is held at DY potential, which in turn is held $200 \mathrm{~V}$ positive relative to the photocathode. In this state the photomultiplier is fully operational. As the gate pulse voltage increases (relative to $F G$ ), the $P$-channel FET will turn off when the pulse reaches between 2.0 and $4.0 \mathrm{~V}$. For pulse voltages of $4.0-6.0 \mathrm{~V}$ both transistors are guaranteed to be off by the manufacturer's specifications. The $N$-channel FET will turn on between 6.0 and $8.0 \mathrm{~V}$ and will be fully conducting when the pulse exceeds $8.0 \mathrm{~V}$. The focusing electrode will then be pulled down to $\mathrm{FG}, 100 \mathrm{~V}$ negative with respect to the PC. Photoelectrons emitted from the cathode will be swept away from the first dynode effectively tuming the $\mathrm{IMT}$ off. In this way there is at least a $2-V$ margin between the point at which the $P$-channel FET turns off and the $N$-channel FET turns on. The opposite is true for the falling edge of the gate pulse. This eliminates the possibility of generating a short circuit to ground on either edge of the pulse.

The totem pole configuration of the MOSFET pair allows the focusing electrode to be actively puiled up to DYI potential or pulled down to $F G$, resulting in fast, symmetrical rise and fall times for the gating pulses. In addition, no current is drawn in either state, so quiescent current consumption is negligible. This is an important consideration considering most high-voltage PMT supplies are only capable of sourcing a few milliamps of current. Zener diodes are placed across the gate and source of the MOSFETs to eliminate the possibility of exceeding the maximum gate-to- source voltages of $\pm 20 \mathrm{~V}$. Even though the gate inputs are kept well below these limits, switching transients and ringing, due to stray inductance, can easily perforate the gatesource insulating layer, causing device failure. With the clamping diodes the MOSFETs are very rugged and dependable devices.

\section{I1. PERFORMANCE AND DISCUSSION}

The performance of the gating circuit was evaluated with a 9816 PMT and dynode biasing network shown in Fig. 3 , where $\mathrm{HV}=-1800 \mathrm{~V}$. Figure 4 shows a schematic diagram of the gate input signal and the PMT response, while Table I lists the gating response data measured in the above configuration.

The speed of the gating circuit is ultimately determined

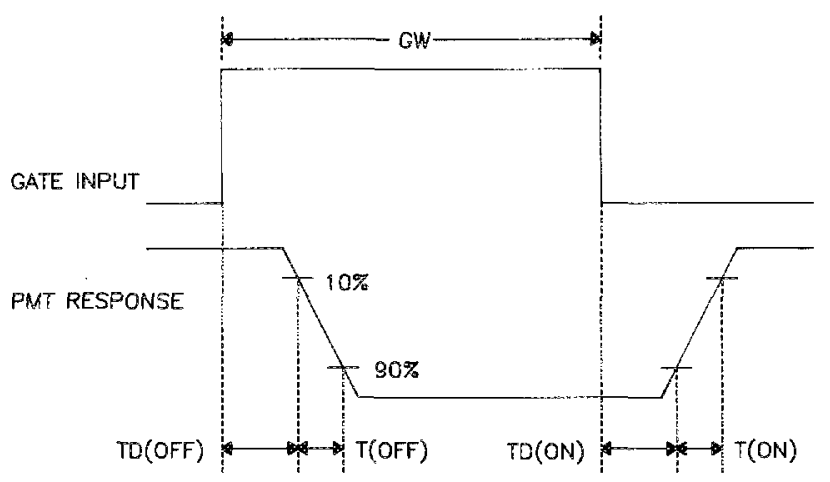

FIG. 4. Schematic of the PMT response relative to the gate signal input. GW is the gate width, TD(of) is the turn-off delay, TD(ori) is the turn-on delay, $T$ (of) is the PMT fall time, and $T($ on $)$ is the PMT rise time. 
TARLE I. Photomultiplier gating circuit response.

\begin{tabular}{|c|c|c|}
\hline Minimum gate width & $\mathrm{GW}(\mathrm{min})^{:}$ & $300 \mathrm{~ns}$ \\
\hline Maximum gate width & $G W(\max )$ & $15 \mathrm{~ms}$ \\
\hline Maximum repetition rate & $\operatorname{RP}(\max )$ & $2 \mathrm{kHz}(\mathrm{at} G W=1 \mu \mathrm{s})$ \\
\hline FE rise time $(300 \mathrm{~V})$ & $T_{\mathrm{FI}}(\mathrm{On})$ & $150 \mathrm{~ns}$ \\
\hline FE fall sime $(300 \mathrm{~V})$ & $T_{\mathrm{FF}}($ (Of $)$ & $300 \mathrm{~ns}$ \\
\hline PMT turn-on time & $T($ on $)$ & $40 \mathrm{~ns}$ \\
\hline PMI turn-off time & $T(\mathrm{of})$ & $60 \mathrm{~ns}$ \\
\hline Tum-on delay & TD(on) & $640 \mathrm{~ns}$ \\
\hline Turn-off delay & TD (off) & $620 \mathrm{~ns}$ \\
\hline Extinction efficiency & & $>5000: 1$ \\
\hline Switching transients & & $2.8 \mathrm{mV}$ into $50 \Omega$ \\
\hline Quiescent current & & $<2 \mu \mathrm{A}$ \\
\hline
\end{tabular}

See Fig. 4 for definitions of terms.

by how fast the focusing electrode can be switched. The focusing electrode voltage has a fall time $\left[T_{\mathrm{FE}}\right.$ (of) $]$ of $300 \mathrm{~ns}$ (turning the PMT off) and a rise time $\left[T_{\mathrm{FE}}\right.$ (on) $]$ of $150 \mathrm{~ns}$ (tuming the PMT on) over its full $300-V$ swing. Because of the sensitivity of PMT gain with respect to the focusing electrode voltage, the actual PMT response is much faster. The PMT turn-off time $\left(T_{\text {off }}\right)$, between $10 \%$ and $90 \%$ extinction, is $60 \mathrm{~ns}$ and the turn-on time $\left(T_{\text {on }}\right)$ is $40 \mathrm{~ns}$, with an overall extinction efficiency of $>5000: 1$. This allows eff. cient pulse rejection and allows measurements to proceed $<100$ as following the pulse. Note also that the response is relatively symmetrical which may be important in situations where, for example, fast gain attenuation is required directly following a trigger signal.

The circuit does exhibit some delays between the initiation of the TTL input pulse and the response of the PMT to the pulse. The turn-off (TD of ) and turm-on (TD ${ }_{\text {un }}$ ) delays of 620 and 640 ns, respectively, are mainly due to propagation delays in the $6 \mathrm{~N} 136$ optocoupler (typically $0.5-1.5 / \mathrm{s}$ ). These delays are highly reproducible from one pulse to another and are easily compensated for with appropriate timing delays. They therefore generally do not interfere with overall gating performance. These delays can be significantly reduced by using a high-speed optocoupler such as a 6N137 (propagation delay typically $45-75 \mathrm{~ns}$ ), although such devices only achieve these higher speeds at the expense of much greater power consumption.

The minimum gate width $\mathrm{GW}(\mathrm{min})$ of $300 \mathrm{~ns}$ is determined by the rise and fall time of the $300-V$ focusing electrode pulse, which is limited by the speed at which the bipolar transistors can charge and discharge the MOSFET input gate capacitance. Decreasing the gate width beyond this limit reduces the full voltage swing of the focusing electrode, degrading extinction efficiency. The maximum gate width $\mathrm{GW}$ (max) of $15 \mathrm{~ms}$ is set by the $50-\mu f$ charge storage capacity of low-voltage MOSFET gate drive circuitry and the current consumption of the optocoupler during an off period.

The only artifacts produced by the gating circuit are switching transients (into $50 \Omega$ ) of $+2.8 \mathrm{mV}$ (PMT turn off) and $-2.8 \mathrm{mV}$ (PMT turn on) that are due to capacitative coupling of the gate pulse into the PMT anode. These transients, measured under no-light conditions, are typical for gating circuits which switch high voltages in close prox-

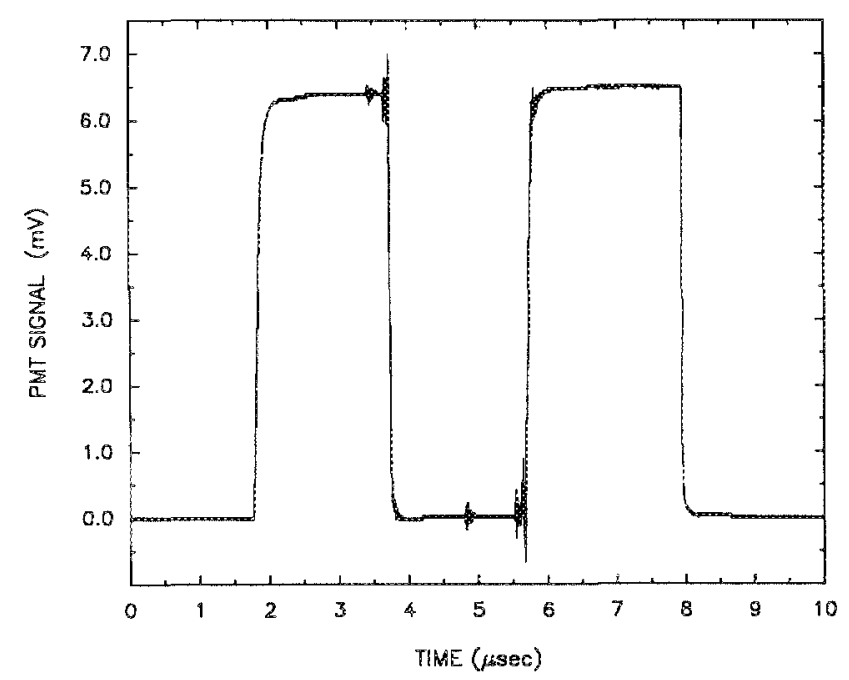

FIG. 5. PMT response to a $6-\mu$ s LED pulse recorded with a Biomation 8100 Transient Recorder. Plotted on the vertical axis is PMT response in millivols (into $50 \Omega$ ) vs time in mieroseconds. The middle of the LED pulse is gated off during a $2-\mu$ s window.

imity to the pickup-sensitive anode. ${ }^{25}$ Manufacturen's specification for the EMI GB1001A report switching transients of $5 \mathrm{mV}$ into $50 \Omega$. The transients observed for our gating circuit are relatively small, very consistent in size and duration from one pulse to another, and independent of changes in the HV input and light intensity. They can therefore be easily subtracted as backgrounds from digitized sample data and do not interfere with light intensity measurements.

Figure 5 shows the PMT response to a LED pulse $6 \mu$ s in duration with the middle portion of the pulse gated of for 2 $\mu s$. The data were collected with a Biomation 8100 transient recorder and plotted as signal intensity in millivolts (into 50 @) versus time in microseconds. The trace shows the fast response, symmetrical rise and fall times, low noise, and high attenuation of photomultiplier response. The slight rounding of the PMT output at the beginning of the LED pulse and the end of the gate window is most likely due to gain fluctuations caused by high currents at the last few dynodes. As current fows through the resistors near the anode the voltage will increase causing a temporary increase in gain. The problem can be minimized by placing zener diodes across the last pair of dynodes to help stabilize the voltage.

Although data reported here was taken with the PMT configured in an analog mode, the gating circuit works equally well in a single-photon pulse counting mode. Studies are in progress using this gating circuit for low-light-level luminescence measurements of translational and rotational diffusion in biological membranes.

\section{ACKNOWLEDGMENTS}

The authors would like to acknowledge Ralph Schliephacke, Wilhelm Streicher, and Karl-Friedrich Lehmann of the Max-Planck Institut für Biophysikalische Chemie for their technical expertise in circuit layout and construction. This work was supported in part by NHH grant A121873 
(B.G.B.), by a Fulbright Senior Fellowship (B.G.B.), and a Proctor \& Gamble Graduate Fellowship (T.M.Y.).

a) Author to whom correspondence should be addressed.

${ }^{\prime}$ R. J. Woods, S. Scypinski, L. J. Cline Love, and H. A. Ashworth, Anal. Chem. 56, 1395 (1984).

2M. Vclez and D. Axelrod, Biophys. J. 53, 575 (1988).

${ }^{3}$ T. M. Yoshida and B. G. Barisas, Biophys. J. 50, 41 (1986).

${ }^{4}$ A. F. Corin, E. Blatt, and T. M. Jovin, Biochemistry 26, 2207 (1987).

${ }^{5}$ D. H. Hartman, Rev. Sci. Instrum. 49, 1130 (1978).

'S. G. Ballard, Rev. Sci. Instrum. 54, 1473 (1983).

${ }^{7}$ S. Singer, L. K. Neher, and R. A. Ruehle, Rev. Sci. Instrum. 27, 40 (1956).

${ }^{8}$ R. G. Bennett and R. P. Schwenker, Rev. Sci. Instrum. 30, 836 (1959).

'R. G. Bennett, Rev. Sci. Instrum. 31, 1275 (1960).

${ }^{10}$ M. L. Bhaumik, G. L. Clark, J. Snell, and L. Ferder, Rev. Sci. Instrum. 36, 37 (1965).
"\$. Minami and K. Nishikawa, Appl. Opt. 5, 173 (1966).

${ }^{12}$ F. De Martini and K. P. Wacks, Rey. Sci. Instrum. 38, 866 (1967).

${ }^{13}$ F. De Marco and E. Penco, Rev. Sci. Instrum. 40, 1158 (1969).

${ }^{14}$ T. D. S. Hamilton, J. Phys. E: Sci. Instrum. 4, 326 (1971).

${ }^{15}$ M. Rossetto and D. Mauzerall, Rev. Sci. Instrum. 43, 1244 (1972).

${ }^{16}$ T. D. S. Hamilton and K. Razi Naqui, Anal. Chem. 45, 1581 (1973).

${ }^{17}$ M. Yamashita, Rev. Sci. Instrum. 45, 956 (1974).

${ }^{[8}$ J. J. Ramirez and L.W. Kruse, Rev. Sci. Instrum. 47, 832 (1976).

19 U. Farinelli and R. Malvano, Rev. Sci. Instrum. 29, 699 (1958),

${ }^{20}$ K. B. Keller and B. M. K. Nefkens, Rev. Sci. Instrum. 35, 1359 (1964).

${ }^{2}$ B. L. Elphick, J. Phys. E 2, 953 (1969).

${ }^{22}$ N. Klein and T. J. Rock, Rev. Sci. Instrum. 41, 1671 (1970).

${ }^{2.3}$ D. G. Jameson and J. J. Martin, J. Phys. E 8, 635 (1975).

${ }^{24}$ B. G. Barisas and M. D. Luther, Rev. Sci. Instrum. 51, 74 (1980).

${ }^{25} \mathrm{R}$. Wardle, Gating of Photomultipliers (Thorn EMI Gencom Inc., NJ, 1982).

${ }^{26}$ M. D. Seltzer, M. S. Hendrick, and R. G. Michel, Anal. Chem. 57, 1096 (1985). 